\title{
Los determinantes sociales y económicos de la salud. Asuntos teóricos y metodológicos implicados en el análisis*
}

\section{Social Determinants of Health. Theoretical and Methodological Issues Involved in the Analysis}

\section{Determinantes sociais e econômicos da saúde. Questões teóricas e metodológicas implicados na análise}

Fecha de recepción: 17-02-14 Fecha de aceptación: 17-03-14 Disponible en línea: 01-07-14

doi: 10.11144/Javeriana.rgyps13-27.dses

Cómo citar este artículo:

Álvarez-Castaño L. Los determinantes sociales y económicos de la salud. Asuntos teóricos y metodológicos implicados en el análisis. Rev. Gerenc. Polít. Salud. 2014; 13(27): 28-40. http://dx.doi. org/10.11144/Javeriana.rgyps13-27.dses 


\section{Resumen}

Los estudios sobre determinantes de la salud son cuestionados por mostrar diferencias en los resultados en salud entre estratos sociales, sin explicitar los referentes doctrinarios de los que parten ni establecer los procesos de determinación que están involucrados en los análisis. Igualmente, se cuestiona que con frecuencia omiten el carácter relacional de los grupos, sectores o clases sociales analizadas. Por esa razón, esta revisión tuvo por objetivo aportar algunos elementos que fundamenten el campo de conocimiento, tanto en sus aspectos teóricos como en los prácticos, los cuales tienen un rol central en cualquier investigación sobre el tema, se hagan explícitos o no. Se analizaron aportes de algunas teorías de justicia social, así como de la epidemiología ecosocial y de estudios sobre estilos de vida provenientes de la sociología médica. Igualmente, se revisaron conceptos básicos sobre estratificación social y posición socioeconómica que son usados recurrentemente en este campo de conocimiento.

Palabras clave: desigualdades en la salud; clase social; equidad en salud; justicia social; inequidad social; Colombia

\section{Abstract}

The studies on health determinants are questioned because they show differences on health results between social strata, without explaining the doctrinaire referents they are based on, or establishing the determination processes involved in the analysis. Likewise, it is brought into question how they frequently omit the relational character of the groups, sectors or social classes being analyzed. For this reason, the objective of this review was to contribute some elements that provide a basis for the knowledge field, both on the theoretical as well as on the practical aspect, which have a central role in any research on this topic regardless if they are explicit or not. We analyzed contributions from some social justice theories as well as eco-social epidemiology and studies on lifestyles coming from medical sociology. Likewise, we reviewed basic concepts on social stratification and socio-economic position that are repetitively used in this field of knowledge.

Keywords: health inequalities; social class; equity in health; social justice; social inequity; Colombia

\section{Resumo}

Os estudos sobre determinantes da saúde são questionados por mostrar diferenças nos resultados em saúde entre camadas sociais, sem explicitar os referentes doutrinários dos quais partem nem estabelecer os processos de determinação que estão envolvidos nas análises. Igualmente, questiona-se que com frequência omitem o caráter relacional dos grupos, setores ou classes sociais analisadas. Por essa razão, esta revisão teve como objetivo aportar alguns elementos que fundamentem o campo de conhecimento, tanto em seus aspectos teóricos quanto práticos, os quais têm um rol central em qualquer investigação sobre o tema, façam-se explícitos ou não. Analisaram-se aportes de algumas teorias de justiça social, assim como da epidemiologia eco social e de estudos sobre estilos de vida provenientes da sociologia médica. Também foram revisados conceitos básicos sobre estratificação social e posição socioeconômica que são usados recorrentemente neste campo de conhecimento.

Palavras chaves: desigualdades em saúde; classe social; equidade em saúde; justiça social; iniquidade social; Colombia 


\section{Introducción}

En el año 2005, la Organización Mundial de la Salud (oms) creó la Comisión de Determinantes Sociales de la Salud (1). Sin duda uno de los hechos recientes más relevantes para la salud pública y la epidemiología, con efectos al menos sobre dos esferas: en primer lugar, en lo que podría denominarse la institucionalidad de la salud pública y la epidemiología $\mathrm{y}$, especialmente, entre aquellas instituciones y personas que actúan alrededor de los organismos multilaterales. La Comisión y su producción teórica $(2,3)$ crearon, al menos en el discurso, la necesidad de mirar un poco más allá de los bastante agotados linderos de los problemas de la pobreza y sus efectos en la salud. Esto también constituyó un nuevo aire para la asfixia que provocó en muchos países, entre ellos Colombia, la agenda que siguió a las reformas neoconservadoras de los sistemas de salud impuesta por el Fondo Monetario Internancional (FMI), el Banco Mundial y las élites nacionales económicas, políticas y técnicas $(4,5)$.

La otra esfera sobre la que recayeron los efectos de la Comisión de Determinantes Sociales de la Salud (CDSs) fue, sin duda, la académica. Posteriormente a la difusión de sus trabajos, hubo y persiste una nueva "ola" de estudios epidemiológicos y de salud pública, que establecen la presencia de gradientes sociales (resultados diferenciados en salud de acuerdo con algún criterio que mide la posición socioeconómica) en las enfermedades crónicas más prevalentes en el interior de los países de altos y medianos ingresos, así como estudios que demuestran desigualdades en salud entre países y regiones. Ahora bien, como lo han mencionado algunos autores, el volumen de evidencia empírica sobre desigualdades en salud contrasta con un escaso nivel de desarrollo teórico. Muchos estudios no explicitan los referentes doctrinarios de los que parten, ni establecen de manera específica los mecanismos de determinación involucrados en los análisis (6-9).

Acogiendo algunas de las críticas mencionadas, se realizó esta revisión temática, con el objetivo de sintetizar elementos teóricos y metodológicos elaborados por autores de diferentes disciplinas, que pueden contribuir a fundamentar los estudios sobre los determinantes sociales y económicos de la salud. El texto está organizado de la siguiente manera: en primer lugar, se retoma la propuesta de objeto de estudio propuesto por la CDSs, pero agregando consideraciones de otros autores que pueden aportar a precisar los conceptos y el qué se estudia cuando se incursiona en los determinantes sociales y económicos de la salud y sus inequidades resultantes. En segundo lugar, se presentan algunos aspectos de las teorías de justicia social y de la teoría epidemiológica ecosocial, que amplían el horizonte sobre cómo interpretar lo que se estudia, cuáles son algunos vínculos del campo con otras áreas del conocimiento como la filosofía, la biología y la sociología médica. En tercer lugar, se hace una revisión sobre la estratificación y la posición socioeconómica, pues estos conceptos, finalmente, se concretan en cómo se miden las inequidades en salud. En la última parte se plantea una síntesis de los aportes de las teorías revisadas.

La lectura de este texto amerita al menos dos precauciones: en primer lugar, este no intenta dar cuenta, ni mucho menos resolver, los interrogantes teóricos que están en juego en el campo de conocimiento, no solo por limitaciones de la autora, sino porque muchos de los problemas que el campo enfrenta obedecen a debates todavía vigentes de las diferentes disciplinas en que se apoya. En segundo lugar, se trata de una mirada panorámica a los fundamentos propuestos, y no de una revisión exhaustiva de cada uno de ellos, aunque sí se proporciona bibliografía básica en caso de que se quiera una mayor profundización. 


\section{Sobre el objeto de estudio}

Aunque, como se mencionó, a partir del trabajo de la cDss de la oms, hay un creciente número de estudios sobre determinantes sociales de la salud, lo cierto es que no siempre hay consenso sobre cuál es el objeto de estudio de este campo de conocimiento. Aquí formulamos la siguiente propuesta, con base en la propuesta de la cDss, pero con algunas precisiones: como lo proponen Solar e Irwin (2), los DSs son un campo de conocimiento que tiene dos facetas: a) los elementos de la estructura social que condicionan la posición que las personas alcanzan en la sociedad y que tienen un efecto directo en la distribución de los riesgos o de la protección de la salud; y b) las inequidades en la situación de salud cuando se comparan grupos, estratos o clases sociales.

En relación con la primera faceta, es ahí donde de manera más específica nos adentramos al campo de la determinación social. Es decir, se estudian aquellas instituciones, normas, arreglos sociales, políticas, propiedad sobre los medios de producción, formas de vivir, maneras de organizar el entorno que provocan una distribución inequitativa del proceso salud-enfermedad. La segunda faceta se refiere a la manera en que esos elementos de la estructura social y de los modos de vida se expresan y se hacen visibles, es decir, las inequidades en salud. Estas últimas tienen, al menos, dos acepciones. En la más técnica y de uso más extendido, implica que se trata de resultados desiguales en la situación de salud cuando se comparan clases, grupos o estratos sociales (10), y en su acepción ética y política, el término asume que esas desigualdades se consideran injustas e intolerables desde un sistema dado de valores $(11,12)$, pero además que son el resultado de la manera en que la sociedad produce y distribuye los bienes sociales que favorecen o afectan la salud (13).
El uso de los conceptos de determinación o determinantes, no deja de ser polémico y merece una consideración especial. La determinación ha sido cuestionada con argumentos muy sólidos desde las ciencias sociales y aun desde las naturales. Desde muchos lugares se ha interrogado el tratar cualquier aspecto de la vida de los seres humanos, ya sea como individuos o en sociedad, como si se tratara de procesos que se reproducen con precisión matemática, según leyes generales inmutables, repetibles y predecibles (14). Esta es una crítica que también se ha formulado al estudio de los DSs (15), que no ha sido respondida exhaustivamente. En respuesta, la mayoría de autores que se refieren al tema plantean que cuando se analiza la influencia de las condiciones sociales y económicas en la salud de la población, no se alude a formas determinísticas mecánicas; se trata más bien de relaciones probabilísticas, que no son unívocas ni bien definidas y, muy especialmente, que no se trata de variaciones cuantitativas $(13,16)$, pero, en todo caso, se reconoce la existencia de unos hechos, es decir, unos efectos sobre la salud derivados de dichas condiciones, que de ninguna manera pueden considerarse arbitrarios o fruto del azar. Con lo que sí se cuenta para responder a la pregunta sobre la determinación, es con evidencia empírica pertinaz y voluminosa, que desde hace varios siglos demuestra esta influencia de la estructura social y económica sobre la distribución de las formas de enfermar y morir.

\section{Algunos fundamentos teóricos para el estudio de los DSS}

Son varios y provenientes de diferentes disciplinas los referentes teóricos en los que se apoya el campo de conocimiento de los Dss. En este texto se desarrollan de manera general solamente tres, reconociendo que no agotan el universo teórico del tema; estos son: a) 
bases provenientes de las teorías de justicia social, b) una perspectiva epidemiológica para desentrañar la manera como deben interpretarse los diferentes niveles de análisis y los aspectos de naturaleza biológica, social y económica que están en juego; y c) aportes desde la sociología médica para encontrar una convergencia que permita analizar los estilos de vida en clave de determinantes sociales de la salud.

\section{Bases desde la justicia social: el derecho a la salud}

Varias teorías de justicia social ofrecen aportes significativos para la interpretación de los Dss, dado que se ocupan de comprender el problema de la igualdad y su relación con la libertad entre los seres humanos (17, 18). Entre ellos se encuentran los aportes ya bastante reconocidos de Amartya Sen, con su teoría del "desarrollo como libertad para desplegar capacidades" (19-23), y especialmente la ampliación que Martha Nussbaum ha hecho al enfoque, al menos en dos aspectos: establecer su conexidad con los derechos humanos y fundamentarlo en la dignidad humana $(23,24)$, que sin duda constituyen una fundamentación moral para la investigación y acción sobre las inequidades en salud.

Este enfoque propone el espacio de las capacidades (oportunidades) para realizar el proyecto de vida que se considera valioso; las capacidades se entienden como la conjugación de habilidades internas con las posibilidades (oportunidades) que la sociedad ofrece para su despliegue. Desde esta perspectiva, los ingresos o la renta no son el único espacio para la evaluación de la igualdad, sino medios para realizar las capacidades. Para Sen y Nussbaum, las capacidades y oportunidades no son intercambiables; por ejemplo, los ingresos, o una determinada renta, no reemplazan la ausencia de otras oportunidades necesarias para la construcción del proyecto de vida valioso que deben disfrutar todos los seres humanos.

En su lista Nussbaum propone varias capacidades relacionadas con la salud, como poder vivir hasta el término de una vida humana de duración normal, no morir de forma prematura, poder mantener una buena salud y disponer de una alimentación adecuada (24). Estas capacidades y otras señaladas por la autora son requisitos indispensables para la dignidad humana y para considerar a una sociedad como verdaderamente justa $(23,24)$.

Además de proponer una lista específica de capacidades como el espacio en que debemos ser iguales, Nussbaum plantea que estas sean la matriz de una concepción de justicia social mínimamente exigible, estructuradas como núcleo de derecho constitucional. Que las capacidades no solo inspiren las políticas sociales y se enuncien como propósitos nacionales, sino que se garantice su seguridad y exigibilidad con instrumentos jurídicos de rango constitucional en el interior de los países y como acuerdos de cooperación mutua en los escenarios multinacionales (24).

La propuesta de Nussbaum, según la cual las capacidades deben constituirse en obligaciones con rango constitucional para los Estados nacionales, y de alguna manera estar en la base de las relaciones internacionales, coincide con la de otros autores que, desde la perspectiva de los derechos humanos, plantean que la realización de los derechos económicos, sociales y culturales (DESC), entre los que se incluyen la salud, la alimentación, la educación, es interdependiente con el ejercicio de los derechos civiles y políticos (25-30). Esta perspectiva ha sido desarrollada a partir de la estructura y el sistema de Naciones Unidas, con apoyo de fuerzas de la sociedad civil y la Academia de muchos países, y se ha convertido en un 
norte teórico y político en muchos campos, entre ellos la salud pública.

\section{Fundamentos teóricos desde el campo de la epidemiología}

El análisis de los determinantes de la salud requiere una teoría epidemiológica que ayude a concatenar los fenómenos sociales, económicos y biológicos que intervienen en los procesos analizados. Un aporte significativo a este propósito es el realizado por la teoría ecosocial, desarrollada desde hace algunos años especialmente por Nancy Krieger (16, 31-34). Según esta autora, uno de los mayores problemas epistemológicos que resultan cuando se busca ampliar la perspectiva de análisis en los procesos salud-enfermedad, es que con frecuencia se busca refugio en las ciencias sociales, exclusivamente, pero de esta manera se omiten los fenómenos biológicos que subyacen a los procesos de salud-enfermedad. La teoría ecosocial recoge los aportes de las teorías ecológicas y sistémicas aplicadas a la salud (31-34), considerando de manera simultánea tres elementos: a) lo ecológico, b) la influencia de la estructura social en la producción de la salud y la enfermedad, y c) las interrelaciones entre los dos primeros (16).

La teoría suscribe una serie de premisas compartidas por los enfoques ecológicos $(35,36)$, según los cuales para entender la relación y el compromiso entre organismos y su medio ambiente, se requiere desarrollar análisis en relación con niveles, espacio y tiempo, además de considerar estos fenómenos en nichos geográficos e históricos determinados.

Uno de los mayores aportes de la teoría es el concepto de corporeidad (embodiment), que sirve de base para explicar, por ejemplo, la manera en que la discriminación sufrida por las mujeres o por algunos grupos étnicos, se convierte en un determinante estructural de la situación de salud. El embodiment sostiene que los seres humanos incorporamos, es decir, integramos en nuestro cuerpo, nuestras experiencias vividas ecológica y socialmente: "Los determinantes de los patrones actuales de la distribución de la enfermedad son exógenos al cuerpo de las personas y no puede ser reducido a lo que se ha denominado 'características innatas'; aunque sin duda la variabilidad individual es un factor a considerar, la evidencia científica ubica la flecha de la causalidad con un mayor peso proveniente desde las condiciones societales hacia las condiciones de salud de las personas" (16).

Aportes desde la sociología médica: los estilos de vida analizados desde los determinantes sociales y económicos

Uno de los aspectos más polémicos en el estudio de los DSs es el de los estilos de vida, ya que la epidemiología hegemónica los ha catalogado, junto con algunas características individuales de tipo biológico, como los principales predictores $\mathrm{y}$, en últimas, responsables de la distribución social de la salud y la enfermedad. Es importante retomar las investigaciones sobre estilos de vida por fuera del ámbito reduccionista y darles un nuevo significado desde la perspectiva de los Dss, pues es imprescindible abordar las dimensiones individuales, así como las culturales y simbólicas que también tienen una influencia en la distribución social de la salud. Los fenómenos que hoy desempeñan un papel determinante en la salud, tales como: la influencia de los conflictos de género y etnia, los cambios en el mundo laboral, las nuevas formas de exclusión social, las nuevas dinámicas de globalización, los efectos de la educación en la salud, etc., se deben leer también en su dimensión simbólica.

William Cockerham, desde el campo de la sociología médica, formula su teoría con base en los postulados de Max Weber y 
Pierre Bourdieu (37-41). Así, plantea la necesidad de establecer una convergencia entre la dimensión individual (agencia) y las condiciones socioculturales (estructura) para el estudio de los estilos de vida. La estructura, según el autor, establece aquellos condicionantes socioculturales, económicos y políticos que restringen el marco de acción que los individuos tienen sobre su vida. La agencia se refiere a la capacidad de los individuos de direccionar (agenciar) su propio proyecto de vida. El marco teórico propuesto por este autor consta entonces de componentes de orden estructural, y otros de orden individual, que dan forma a los estilos de vida. En relación con los primeros, destaca a la clase social (que es la condición más determinante, pero no la única), al igual que a los condicionantes impuestos por el género y la etnia. Las colectividades también se consideran estructurales, ya que se ha encontrado que la pertenencia a ciertos grupos: religiosos, culturales o políticos, imprime unos estilos de vida particulares en salud. En el campo de la agencia, es decir, en el nivel individual, el autor señala a los escenarios y procesos de socialización, como elementos que dan forma a los estilos de vida en salud. La socialización primaria, es decir, las normas y costumbres aprendidas en la familia, y la socialización secundaria: lo aprendido en la escuela y el trabajo, así como la experiencia personal, son fundamentales pues las personas evalúan y transforman sus comportamientos con base en lo vivido y en los valores y creencias aprendidas en estos espacios. En síntesis, la estructura determina las oportunidades de vida (life chances), que en interacción con las decisiones tomadas por las personas (life choices) crean y predisponen hacia ciertos estilos de vida en salud (41). La convergencia planteada por Cockerham constituye un aporte significativo para incorporar la dimensión cultural e individual desde la perspectiva de los determinantes de la salud.

\section{La estratificación socioeconómica y sus implicaciones en el campo de conocimiento de los DSs}

El aspecto más crítico en el estudio sobre inequidades en salud es la definición teórica y sus repercusiones metodológicas, de las categorías de estratificación social utilizada, que refleja la manera como el investigador interpreta la posición socioeconómica y sus efectos en la distribución de la salud. Con frecuencia, se cuestiona que en los estudios sobre inequidades en salud haya ausencia de definiciones explícitas sobre los grupos, estratos o clases en comparación. Según algunos autores, hay una omisión, pareciera que deliberada, de referencias específicas al carácter relacional de los grupos comparados, relación que se deriva de la posición que ocupa cada uno de ellos en la producción y/ o en la distribución de los bienes sociales $(7,13)$.

La intención en esta parte del texto no es formular una propuesta de estratificación social para el análisis de las inequidades en salud; se trata de un ejercicio mucho más modesto de sintetizar algunas reflexiones que, desde las dos escuelas sociológicas clásicas y desde la epidemiología y la salud pública, contribuyen a explicitar los supuestos que están detrás de los análisis sobre las categorías y los indicadores que se usan para demostrar la distribución inequitativa de los factores que protegen o deterioran la salud.

Aunque en la mayoría de investigaciones no se alude a los criterios para definir la estratificación socioeconómica, lo cierto es que las categorías disponibles en este terreno son las provenientes de las escuelas sociológicas clásicas: el marxismo y la de Max Weber. La primera fue el soporte teórico de muchos de los estudios sobre la determinación social de la salud realizados hasta 1990 , y en ese entonces eran frecuentes los análisis de la 
determinación y la distribución de los procesos de salud y enfermedad, aludiendo a la comparación de resultados en salud como fruto de la confrontación por recursos entre clases sociales y a la explotación de una clase por otra $(7,13)$.

Como se sabe, de manera muy sintética, el marxismo clásico postula que la estructura de clase es producto únicamente de las relaciones derivadas de una dimensión de la vida social: la producción de los bienes y medios materiales de subsistencia, que en las sociedades capitalistas se caracteriza por generar una división del trabajo en la que una clase produce los bienes y la otra clase se apropia del excedente (42). Estas dos clases tienen un carácter eminentemente relacional, interconectadas por una forma concreta de relación que es la explotación (42).

En las últimas tres décadas, la corriente llamada marxismo analítico o neomarxismo ha adaptado la estructura analítica clásica a los cambios producidos en las sociedades capitalistas modernas, tales como la consolidación de las clases medias y la emergencia de otros conflictos, fundamentalmente los de género y de etnia, que claramente se diferencian en su naturaleza de los conflictos producidos por el sistema capitalista. Tal vez el autor más destacado de esta corriente sea Erik. O. Wright, cuyo trabajo se centra en el análisis de las clases medias de hoy, conservando las bases de estructura de clases del marxismo clásico. Para este autor, lo que sucede con las clases medias es que en algunos casos se configura una posición contradictoria de clase según la cual, algunas posiciones están simultáneamente en dos o más clases, como sucede por ejemplo con quienes ejercen funciones directivas en las empresas. Estas posiciones, de manera simultánea, venden su fuerza de trabajo, lo cual las ubica en la clase obrera, pero al mismo tiempo dominan a otros trabajadores en la producción
(42, 43). Wright elaboró una propuesta de estructura de clases que conserva la división de acuerdo con la propiedad de los medios de producción, es decir, sigue habiendo dos clases relacionadas por la explotación, pero se incluye la propiedad de otros bienes, como los organizativos y las habilidades, que generan nuevas categorías entre los asalariados. En total, esta propuesta incluye doce categorías de clasificación, tres corresponden a la clase propietaria de los medios, de acuerdo con la extensión de esa propiedad y nueve corresponde a los asalariados de acuerdo con sus habilidades y recursos organizativos (44).

Con respecto al análisis sobre género y etnia, aunque no es posible establecer generalizaciones, dado que el llamado neomarxismo no es un grupo homogéneo, según Val Burris, en general se aprecia un consenso en la mayoría de los autores contemporáneos en el sentido que los conflictos de clase no subsumen a los derivados del patriarcado y de la discriminación étnica. Lo que sí se evidencia en la literatura marxista es que la relación, el estatus entre estos diferentes conflictos, aún está en discusión: "los expertos conceden un grado importante de autonomía a las formas no clasistas de opresión, aunque aún hay debates sobre el grado de autonomía y su relación con la estructura de clase" (45).

La otra perspectiva teórica sobre la estratificación es la basada en la escuela de Max Weber. Este autor, si bien reconoce la existencia de clases sociales, no lo hace de un modo definitivo como en el marxismo. Antes bien, sostiene que las "clases" no son la única forma de abordar la división de la sociedad. Puede postularse, paralelamente, la división en estamentos y partidos, según se trate de la distribución del poder económico, social o político. Es decir, hay tres generadores de estratificación: la clase, el estatus y el poder (46). Con este modelo de estratificación tridimensional, Weber "autonomiza" las 
esferas económica, social y política y rechaza la posibilidad de adjudicar a una de ellas la determinación en última instancia, relativizando así la importancia primordial otorgada por la teoría marxista a la división de la sociedad en clases (46). Junto a la división en clases, sostiene una concepción multidimensional en la cual las relaciones de clase se entrecruzan con otras bases (no clasistas) de asociación, básicamente el estatus.

Para Weber la posición de clase no está determinada por las relaciones de producción. Estas generan una forma de estratificación pero no la única, ni la más importante. El factor central en la estratificación es la posición en el mercado, es decir, en el espacio de la distribución de los bienes producidos, el cual genera grupos o estratos que comparten una serie de valores, circunstancias u oportunidades de vida (life chances) (45). Por eso, para Weber no hay solo dos clases sociales, hay tantas clases en una sociedad como tipos de capacidades de mercado (43).

Coherente con su fundamentación weberiana, Goldthorpe propuso una clasificación de las clases sociales, fundamentado en las relaciones económicas y específicamente en las relaciones de empleo para el análisis de las sociedades contemporáneas. Así, contempla siete categorías de acuerdo con las características del empleo en tres aspectos: fuentes, seguridad económica y posibilidades o perspectivas de mejora. El esquema está pensado para las sociedades europeas y ha tenido un uso importante en la investigación social y en salud $(44,47)$.

\section{La estratificación socioeconómica y la me- dición de las inequidades en salud}

Según Lynch, en teoría, la escogencia de una medida o clasificación debería depender de cómo considera el investigador que la posición socioeconómica afecta o protege la salud (48). Si bien esto sería lo teóricamente plausible, también es cierto que en ocasiones la definición conceptual se topa con los límites puestos por las posibilidades reales ofrecidas por la información disponible o por la que se puede recolectar.

Este mismo autor sostiene que de estas tradicionales escuelas de pensamiento y su aplicación específica al campo de conocimiento de las inequidades en salud, se deriva que el énfasis individualista de la escuela de Weber ha llevado a la mayoría de los investigadores en epidemiología a usar indicadores de "oportunidades de vida" (life chances) como educación, ocupación e ingreso. La asunción aquí es que los mecanismos vinculados a aspectos de la distribución son los más importantes para la salud, es decir, son las habilidades, los recursos de los individuos, los que forman el vínculo entre la estratificación social y su situación de salud.

Lynch $(48)$ y otros autores $(49,50)$ formulan una propuesta para establecer cuáles son los constructos que se están midiendo cuando son usados los tradicionales indicadores de educación, ocupación e ingresos. Los autores hacen un llamado para no olvidar que, en cualquier caso, se trata de indicadores de naturaleza individual, derivados de condiciones sociales, económicas y políticas estructurales que no están asignadas al azar $(48,49)$. Comprender el contenido de esos indicadores, contribuye a sustentar su significado y el sentido de su uso en el estudio de las inequidades en salud (51-53).

Lynch plantea que el nivel de educación representa la transición de una posición socioeconómica mayoritariamente recibida de los padres a una lograda como adulto, aunque en ocasiones, lo que reflejan las oportunidades educativas realmente, es la posición de los padres. La educación ha demostrado ser un fuerte determinante de la situación de salud, 
especialmente para las mujeres de países de desarrollo medio y bajo. Igualmente, puede reflejar éxitos futuros, en la medida que altos niveles educativos predicen mejores ingresos, viviendas en mejores condiciones y empleos con menor riesgo ocupacional. Aspectos todos vinculados a la distribución de la salud y la enfermedad (48-50). Sin embargo, hay que tener en cuenta que el retorno económico de los recursos invertidos en educación es menor para las mujeres y para algunos grupos étnicos. Igualmente, el número de años ganados en una etapa de la vida informa poco sobre la calidad de la educación recibida (48).

Respecto a la ocupación como indicador de posición socioeconómica, Lynch plantea que, sin duda, las consecuencias para la salud producto de ambientes laborales específicos, es la relación más estudiada desde hace varios siglos. No solo se han analizado los riesgos de trabajos ejercidos en algunas industrias determinadas, sino que también se han establecido las variaciones en las condiciones de salud entre diferentes niveles de trabajadores, como por ejemplo entre los administrativos y los operarios, aunque existan diferentes niveles de ingresos en el interior de esos grupos. Según Lynch, entender la relación entre ocupación y salud constituye la más obvia, evidente e íntima conexión entre los humanos y el proceso productivo que domina nuestra vida de adultos (48).

El trabajo es el vínculo más estructural entre la educación y el ingreso. En los últimos años, las investigaciones sobre el tema han postulado que la relación entre ocupación y salud descansa sobre tres pilares: la demanda psicológica que el oficio ejerce sobre quien lo ejecuta, el grado de decisión y maniobra que la persona tiene sobre su oficio y el soporte social que recibe para su ejecución. Investigaciones realizadas en diferentes contextos han demostrado los efectos psi- cosociales que tiene sobre la salud de los trabajadores el ejercicio de un empleo con amplias demandas, bajo control y bajo nivel de compensación (48).

En relación con el ingreso y la salud, Lynch plantea que es una medida muy útil de posición socioeconómica, porque da cuenta de los recursos materiales que se pueden obtener y que están relacionados con la salud: el ingreso permite adquirir la alimentación, los servicios públicos, la vivienda y otros bienes, cuya ausencia se ha demostrado que deteriora la salud. Pero el autor hace un llamado interesante en términos de los cambios sufridos en el último siglo. Plantea que es necesario adicionar al análisis, ciertas condiciones que él denomina neomateriales, que van más allá de la relación entre salud y pobreza. Es decir, el aumento en el ingreso permite ya no solo satisfacer las necesidades básicas, sino que agrega un conjunto de bienes como calidad en la alimentación, medio ambiente propicio para la actividad física, vivienda libre de condiciones climáticas extremas, que adicionan ventajas para la salud (49).

\section{A manera de síntesis}

A pesar de que el trabajo de la cDss de la oms propició un nuevo aire para el análisis de las relaciones entre la estructura social y las inequidades en salud, muchos de los estudios realizados adolecen de referentes teóricos que los fundamenten y de una mención del carácter relacional de los grupos o estratos que están siendo comparados. Esta ausencia limita el avance conceptual y metodológico del campo de conocimiento, así como sus alcances en la formulación de políticas en salud. Diferentes disciplinas aportan referentes conceptuales a la tarea de fundamentar los estudios sobre la determinación social de la salud y las inequidades resultantes. En este texto se presentan algunas de ellas. 
En primer lugar, se afirma que una vida libre de enfermedades evitables y con una duración por lo menos similar al conjunto de la sociedad, así como libre de hambre, debería ser un derecho consagrado constitucionalmente y realizado en las políticas sociales. A fundamentar esta perspectiva se han dedicado algunas teorías de justicia social, así como activistas sociales y académicos. Ella sin duda constituye la inspiración para encuadrar la relevancia social del tema y la pertinencia de su reivindicación política. Pero, además, se requiere un cambio en la manera de analizar las causas y las manifestaciones de la distribución desigual de la salud y la enfermedad, incorporando las dimensiones biológicas y su interacción con las condiciones sociales, en una perspectiva sistémica y ecológica a lo largo de la vida de los seres humanos. De esta manera, tal como lo propone la epidemiología ecosocial, se rompen los límites de las explicaciones meramente biológicas o exclusivamente sociales.

Asimismo, es necesario repensar el lugar de los estilos de vida para superar el lugar preponderante que la epidemiología hegemónica tradicional les ha otorgado. Una convergencia entre el papel de la estructura social con la capacidad de agencia de los individuos y el papel de sus espacios de socialización, permite una ampliación del horizonte de análisis, para no caer en la dicotomía individuo-sociedad. Igualmente, es imprescindible una postura del investigador sobre el tipo de relación entre los grupos, clases o estratos que analiza, aun considerando las limitaciones impuestas por la disponibilidad de información. Finalmente, es importante destacar que cualquier "factor" o "variable" no puede ser considerado un determinante social de la salud. Su escogencia debe inspirarse en una postura sobre cómo se mide la posición socioeconómica y cuál es el lugar en la distribución del proceso salud-enfermedad.

\section{Agradecimientos}

La autora reconoce el apoyo de la estrategia de sostenibilidad de la Universidad de Antioquia 2014-2015 para el desarrollo de este texto.

\section{Referencias bibliográficas}

1. World Health Organization. Social Determinants of Health; 2013 [Internet] [acceso: 12 de junio del 2013]. Disponible en: http://www.who.int/social_determinants/thecommission/en/

2. Commission on Social Determinants of Health. A Conceptual Framework for Action on the Social Determinants of Health; 2007 [Internet] [acceso: 12 de junio del 2013]. Disponible en: http://www. who.int/social_determinants/resources/csdh_framework_action_05_07.pdf

3. World Health Organization (wHo). Improving Equity of Health by Addressing Social Determinants. Génova: wHo Library Catalogu ing-inPublication Data; 2011.

4. Banco Mundial. Informe sobre el Desarrollo Mundial 1993: Invertir en salud. Washington D. C: Banco Mundial; 1993.

5. Cornia G, Jolly J, Stewart F. Adjustment with a Human Face. Oxford: Clarendon Press, World Bank; 1987.

6. Raphael D. Social Determinants of Health: Present Status, Unanswered Questions, and Future Direction. Int J Health Serv. 2006; 36 (4): 651-77.

7. Muntaner C, Rocha K, Borrell C, Vallebuona C, Ibáñez C, Benach J et ál. Clase social y salud en América Latina. Rev Panam Salud Pública. 2012; 31(2): 166-75.

8. Breilh J. La determinación social de la salud como herramienta de transformación hacia una nueva salud pública (salud colectiva). Rev Fac Nac Salud Pública. 2013; 31(Supl 1): S13-S27.

9. Rubio DC. Causalidad, derechos humanos y justicia social en la Comisión de Determinantes Sociales en Salud. Rev Fac Nac Salud Pública 2013; 31(Supl 1): S87-S90.

10. Whitehead M. The Concepts and Principles of Equality and Health. Copenhague: wHo Regional Office for Europe; 2000.

11. Hernández M. Desigualdad, inequidad e injusticia en el debate actual en salud: Posiciones e implicaciones. En: Eibenschutz Hartman C, Tamez González S, González Guzmán R. ¿Determinación social o determinantes sociales de la salud? México D. F: Universidad Autónoma Latinoamericana; 2011. pp. 169-94.

12. Almeida-Filho N. A problemática teórica da determinacao social da saúde. En: Passos Nogueira $\mathrm{R}$, organizador. Determinacao social da saude e reforma sanitária. Río de Janeiro: Cebes; 2010. pp. 13-36. 
13. Breilh J. Epidemiología crítica, ciencia emancipadora e interculturalidad. Buenos Aires: Lugar Editorial; 2003.

14. Mardones JM. Filosofía de las ciencias humanas y sociales: Materiales para una fundamentación científica. Barcelona: Anthropos Editorial; 2001.

15. Peñaranda F. Salud pública y justicia social en el marco del debate determinantes -determinación social de la salud. Rev Fac Nac Salud Pública 2013; 31 (1): S91-S102.

16. Krieger. N. Epidemiology and the People'S Health. Theory and Context. Oxford: Oxford University Press; 2011. pp. 163-201.

17. Rawls J. Una teoría de la justicia. México D. F: Fondo de Cultura Económica; 1997.

18. Gracia D. Fundamentos de bioética. Madrid: Eudema; 1989.

19. Sen A. Desarrollo y libertad. Santafé de Bogotá: Planeta; 1999.

20. Sen A. Nuevo examen de la desigualdad. Madrid: Alianza Editorial; 1995.

21. Sen A. Sobre ética y economía. Madrid: Alianza Universidad; 1997.

22. Sen A, Nusbaumm M. La calidad de vida. México D. F: Fondo de Cultura Económica; 1996.

23. Nussbaum M. las fronteras de la justicia. Consideraciones sobre exclusión. Barcelona: Paidós; 2007.

24. Nussbaum M. Crear capacidades. Propuesta para el desarrollo humano. Barcelona: Paidós; 2012.

25. Defensoría del Pueblo, ProSeDher. El derecho a la salud en la Constitución: la jurisprudencia y los instrumentos internacionales. Bogotá D. C: Defensoría del Pueblo, ProSeDher; 2003.

26. Pautassi L. Los difusos vínculos de articulación entre las políticas públicas y los derechos humanos. En: Vivero JL, Erazo X, editores. Derecho a la alimentación. Políticas públicas e instituciones contra el hambre. Chile: Lom ediciones, Fundación Henry Dunant, Iniciativa América Latina y el Caribe sin hambre; 2009. pp. 11-39.

27. Vivero JL, Ramírez P. Políticas públicas e instituciones contra el hambre. En: Vivero JL, Erazo $\mathrm{X}$, editores. Derecho a la alimentación. Políticas públicas e instituciones contra el hambre. Chile: Lom ediciones, Fundación Henry Dunant, Iniciativa América Latina y el Caribe Sin Hambre; 2009. pp. 41-75.

28. Quintero Mosquera DP. La salud como derecho: Estudio comparado sobre grupos vulnerables. Bogotá: Siglo del Hombre Editores, Universidad Icesi, Red Alas; 2011.

29. Echeverri López E. Derecho a la salud, estado y globalización. Rev Fac Nac Salud Pública. 2006 Mar; (24): 80-95.

30. Echeverry López ME. La acción de tutela en salud: una herramienta para la construcción del derecho a la salud como derecho fundamental. En: Parra Vera Ó, Arango Castrillón A, Gómez Ceballos D, Nieto López E, Noguera Montezuma P, Paja Becoche R, Rodríguez Tejada CM, Echeverry López $\mathrm{ME}$, editores. Indignación justa: estudios sobre la acción de tutela en salud en Medellín. Medellín: Universidad de Antioquia; 2013.pp. 43-08.
31. Krieger N. Theories for Social Epidemiology in the $21^{\text {st }}$ Century: An Ecosocial Perspective. Int $\mathrm{J}$ Epidemiol. 2001; 30 (4): 668-77.

32. Krieger N. Embodyng Inequality. Epidemiologic Perspectives. Amityville, NY: Baywood Publishing Company; 2004.

33. Koch T. Social Epidemiology as Medical Geography: Back to the Future. GeoJournal. 2009; 74: 99-06.

34. Krieger N. Putting Health Inequities on the Map: Social Epidemiology Meets Medical/Health Geography - An Ecosocial Perspective. GeoJournal. 2009; 73 (2): 87-97.

35. Samaja J. Epistemología de la salud: Reproducción social, subjetividad y transdisciplina. Buenos Aires: Lugar Editorial; 2004.

36. Almeida Filho N. Complejidad y transdisciplinariedad en el campo de la salud colectiva: Evaluación de conceptos y aplicaciones. Salud Colect. 2006; 2 (2): 123-46.

37. Cockerham W. Medical Sociology, undécima edición. Nueva Jersey: Prentice Hall; 2009.

38. Cockerham W, Rütten A, Abel T. Conceptualizing Contemporary Health Lifestyles: Moving beyond Weber. The Sociological Quarterly. 1997; 38 (2): 321-42.

39. Bourdieu P. La distinción: Criterios y bases sociales del gusto. Madrid: Taurus; 1988.

40. Christensen VT, Carpiano RM. Social Class Differences in BMI among Danish Women: Applying Cockerham's Health Lifestyles Approach and Bourdieu's Theory of Lifestyle. Soc Sci Med. 2014; 112: 12-21.

41. Abel T, Frohlich KL. Capitals and Capabilities: Linking Structure and Agency to Reduce Health Inequalities. Soc Sci Med. 2012; 74 (2): 236-44.

42. Wright EO. ¿Qué tienen las clases en común para ser clases? En: Vega R, editor. Marx y el siglo xxI: Hacia un marxismo ecológico y crítico del progreso. Bogotá, D. C: Editorial Antropos; 1998. pp. 31-51.

43. Wright EO. Reflexionando una vez más sobre el concepto de estructura de clases. En: Carabaña J, de Francisco A, compiladores. Teorías contemporáneas de las clases sociales. Madrid: Pablo Iglesias; 1995. pp. 17-126.

44. Bergman M, Joye D. Comparing Social Stratification Schemas; 2001 [Internet] [acceso: 13 de junio del 2013]. Disponible en: http://www.freewebs.com/ stratificare_mobilitate/Comparing $\% 20$ Social $\% 20$ Stratification $\%$ 20Schemas.pdf

45. Burris V. La síntesis neomarxista de Marx y Weber sobre las clases. En: Carabaña J, de Francisco A, compiladores. Teorías contemporáneas de las clases sociales. Madrid: Pablo Iglesias; 1995. pp. 127-56.

46. Duek C, Inda G. La teoría de la estratificación social en Max Weber: Un análisis crítico. Revista Austral de Ciencias Sociales. 2006; 11: 5-24.

47. Goldthorpe J, Mknight A. The Economic Basis of Social Class. London: Centre for Analysis of Economic Exclusion; 2004 [Internet] [acceso: 13 de junio del 2013]. Disponible en: http://eprints.lse.ac.uk/6312/1/ The_Economic_Basis_of_Social_Class.pdf 
48. Lynch J, Kaplan G. Socioeconomic Position. En: Berckman L, Kawachi I, editores. Social Epidemiology. Oxford: Oxford University Press; 2000. pp. 13-45.

49. Galobardes B, Shaw M, Lawlor D, Smith GD, Lynch J. Indicators of Socioeconomic Position: Methods in Social Epidemiology. J Epidemiol Community Health. 2006; 60: 47-85.

50. Khang YH, Lynch JW, Yang S, Harper S, Yun SC, Jung-Choi K, Kim HR. The Contribution of Material, Psychosocial, and Behavioral factors In Explaining Educational and Occupational Mortality Inequalities in a Nationally Representative
Sample of South Koreans: Relative and Absolute Perspectives. Soc Sci Med. 2009; 68 (5): 858-66.

51. Kawachi I. Income Inequality and Health. En: Berkman L, Kawachi I, editores. Social Epidemiology. Oxford: Oxford University Press; 2000. pp. 76-94.

52. Harper S, Lynch J. Measuring Health Inequalities. En: Oakes M, Kaufman JS. Methods in Social Epidemiology. San Francisco: Jossey-Bass; 2006. pp. 134-68.

53. Macintyre S, McKay L, Der G, Hiscock R. SocioEconomic Position and Health: What You Observe Depends on How You Measure it. J Public Health Med. 2003; 25 (4): 288-94. 